\title{
Title: Design and development of self-healing dental composites
}

George Huyang, Anne E. Debertin, and Jirun Sun*

Dr. Anthony Volpe Research Center, American Dental Association Foundation, National Institute of Standards and Technology, 100 Bureau Dr., Gaithersburg, MD 20899, USA

*Corresponding Author: Jirun Sun

100 Bureau Drive, Stop 8546

Gaithersburg, MD 20899-8546

Phone: (+1) 301-975-5439

Fax: (+1) 301-963-9143

E-mail: jsun@nist.gov 


\begin{abstract}
:
The purpose of this project is to design and develop a clinically applicable self-healing dental composite (SHDC). The value of resin-based dental restorations could be improved by increasing their service lives. One way to improve longevity is to obturate micro-cracks that form during or after the composite hardens in the dental cavity. Toward this end, we introduce here a new type of SHDC made with contemporary dental components plus two additional ingredients: a healing powder (HP, strongtium fluoroaluminosilicate particles) and a healing liquid (HL, aqueous solutions of polyacrylic acids) that is enclosed within silica microcapsules. As micro-cracks develop, they will break the microcapsules in their propagation path, thereby releasing HL. This liquid will then react with particles of HP exposed by the crack formation, forming an insoluble reaction product that fills and seals the cracks. The key factors to achieve this self-healing of cracks are discussed. The elastic modulus of a SHDC appeared to be satisfactory. The healing process was confirmed by means of mechanical, morphological, and chemical methods. The SHDC restored micro-cracks without external intervention, thereby showing potential for increasing the service lives of dental restorations. Importantly, this SHDC contains only clinically-tested, biocompatible materials, making it readily applicable.
\end{abstract}

Keywords: self-healing, composite, dental material, encapsulation 


\section{Introduction}

Self-healing mechanisms are biomimetic models [1-6] of autonomic repair systems in living tissues that efficiently handle damage, for example, the healing of a broken bone. Inspired by natural biological systems, continuous efforts are being made to mimic natural materials and integrate self-healing capabilities into polymers and polymer composites. Selfhealing has become one of the most desired properties in material development since the first autonomous crack-repair material was synthesized [7-14] by groups at University of Illinois at Urbana-Champaign (UIUC). The progress has opened a new era of intelligent materials. [8, 15-17] Autonomic self-healing composites have shown significant enhancement in extending the service life of polymeric materials. [18, 19]

Dental resin composites play significant roles in a variety of dental applications, including, but not limited to, restorative materials, cements for single or multiple tooth prostheses and orthodontic devices, inlays, onlays, cores and buildups, root canal posts, and provisional restorations. [20] Micro-cracking induced by thermal and mechanical fatigue is a longstanding problem in dental resin composites. They are hard to detect, and almost impossible to repair manually. If left untreated, this fatal deterioration leads to catastrophic failure of restorations, and hence significantly shortens the duration of their service lives. [8] Progress was made to repair cracks in dental resins with use of the monomer-catalyst self-healing model, [21-23] but concerns were raised regarding the potential toxicity of the monomers and the price of the Grubbs catalyst. Also, polyurethane microcapsules are easily fractured when being mixed with silica particles due to the big contrast in their physical strengths.

Herein, we present our design and development of a new model of self-healing dental composites (SHDCs). This model will be achieved by combining the fracture-release-heal process in the UIUC self-healing model [8] with use of glass ionomer cements (GICs), dental restorative materials in current clinical use. [24-26] These two technologies are successful individually but have never been evaluated together. Therefore, we design to replace the 
potential toxic and expensive healing agents in the UIUC model with clinically acceptable GICs. Conventional dental composites [27, 28] generally comprise two key components: 1) dimethacrylate-based polymers to provide a resin network, 2) reinforcing filler particles treated with coupling agents to bind the resin to the particles. In contrast, the SHDCs have two additional components: (1) healing powder (HP): strontium fluoroaluminosilicate particldes, and (2) healing liquid (HL): aqueous solutions of polyacrylic acids encapsulated in silica microcapsules. The microcapsules will protect the HL from premature release during composite preparation. Upon cracking, the HL will be released from microcapsules and react with HP upon contact to form a reparative GIC within the crack. This material is a mixture of reacted polyacrylate salts and unreacted silica gel, and will become physically integrated with other fillers inside the composite matrix. Water is added to draw out HL into the crack, and also simulate hydration from the oral environment. The three distinctive self-healing steps are illustrated in Figure 1: when a crack is forming, and water comes in (A), the microcapsule breaks and releases the HL (B), which then dissolves the HP and forms the GIC (C) that repairs the defect.

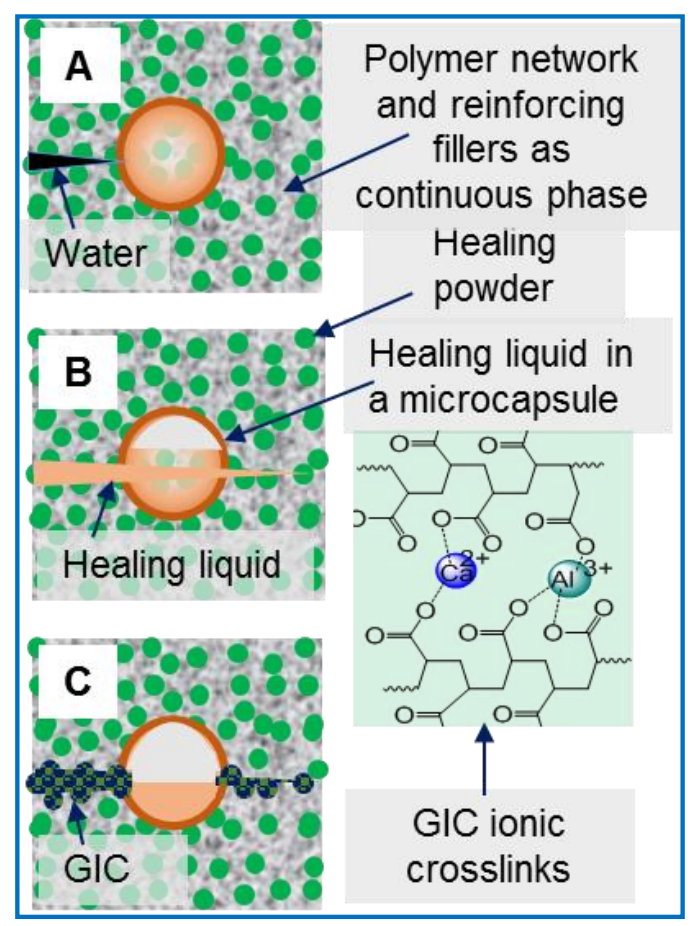


Figure 1. Self-healing steps of SHDC. (A) A crack forms, and water comes in; (B) a microcapsule is broken due to crack propagation and HL is released; $(\mathbf{C})$ the HL reacts with $\mathrm{HP}$, and the product is GIC with ionic crosslinking network.

Based on this model, we formulated and prepared a series of SHDCs with different mass percentages of silica microcapsules containing HL. With use of these SHDCs, we demonstrated the autonomic crack repairing of SHDCs in simulated oral environments based on mechanical, chemical, and morphological evidences. In addition, the encapsulation of HL in silica microcapsules, the effects of microcapsule silanization in the self-healing process, and the mechanical performance of the SHDC in terms of elastic modulus and fracture toughness will be discussed.

\section{Materials and Methods}

Materials: The resin monomers 2-bis(4-(2-hydroxy-3-methacryloxypropoxy)phenyl)propane (Bis-GMA) and hydroxyethyl-methacrylate (HEMA) were supplied by Esstech Inc (Essington, PA, USA). The glass ionomer cement powder and HL were purchased under the product name Riva Protect from SDI Ltd (Bayswater, VIC, Australia). All other reagents and solvents were purchased from Sigma-Aldrich (St. Louis, MO, USA) and used without treatment.

Micro-encapsulation of $H L$ : HL-containing microcapsules were prepared using a silica condensation method. [29, 30] A mixture of $4.0 \mathrm{~mL}$ SPAN 80 surfactant, $100 \mathrm{~mL}$ decahydronaphthalene, $4.0 \mathrm{~mL}$ milli-Q water, and $4.0 \mathrm{~mL}$ polyacrylic acid HL was prepared as the water/oil emulsion. A separate silica mixture containing $4.0 \mathrm{~mL}$ TEOS and $1 \mathrm{~mL}$ hydrochloric acid (2 M) was mixed separately. After 1 hour, the silica mixture was added dropwise into the water/oil emulsion, and the combined mixture was stirred at 400 RPM for 1 hour. After 1 hour, initial signs of precipitation was observed, and the stirring was continued with heating to $60{ }^{\circ} \mathrm{C}$ for 45 minutes. The resulting precipitate was collected by filtration and washed 3 times with ethanol to remove excess SPAN 80 surfactant. The precipitate formed was confirmed by SEM to have microcapsule structure. 
Characterization of microcapsules and encapsulation of HL: Microcapsules were observed on a stereo optical microscope (Leica MZ16, Leica Microsystems, Heerbrugg, Switzerland) and a SEM (JSM-5300, JEOL USA Inc., Peabody, MA, USA) after coating with gold using a Desk V HP (Denton Vacuum LLC., Moorestown, NJ, USA) sputter system. The FTIR measurements of microcapsules were carried out in the Nexus 670 FTIR spectrophotometer (Thermo Scientific, Madison, Wisconsin, USA). Microcapsules were crushed and mixed with $\mathrm{KBr}$ powder (3 mg AP25 in $300 \mathrm{mg} \mathrm{KBr}$ ) and pressed into pellets. A total of 64 scans were collected from $650 \mathrm{~cm}^{-1}$ to $4000 \mathrm{~cm}^{-1}$ at $4 \mathrm{~cm}^{-1}$ resolution. The wt $\%$ of water and HL within the microcapsules was measured using thermogravimetric analysis (Q500, TA Instruments, New Castle, DE, USA), with the temperature raised step-wise to $150{ }^{\circ} \mathrm{C}$ for $1 \mathrm{~h}, 400{ }^{\circ} \mathrm{C}$ for 1 $\mathrm{h}$, and $600{ }^{\circ} \mathrm{C}$ for $1 \mathrm{~h}$ under a nitrogen atmosphere, and then to $1000{ }^{\circ} \mathrm{C}$ under a normal atmosphere.

Silanization of the microcapsules: The microcapsules were surface treated with silanization to give them strong surface binding with the methacrylate resins used in the composite, to ensure that the microcapsules break successfully as the composite breaks. The microcapsules were added to a solution of $20 \mathrm{~mL}$ of hexane, $200 \mu \mathrm{L}$ of 3-methacryloxypropyltrimethoxy silane and $20 \mu \mathrm{L}$ of formic acid (88\%), and stirred for 5 minutes. The microcapsules were recollected by filtration and washed 3 times with hexane.

Construction of the SHDC: The B/H resin mixture used for the SHDC were made with BisGMA (49.5 \%), HEMA (49.5 \%), camphorquinone $(0.5 \%)$ and ethyl 4(dimethylamino)benzoate $(0.5 \%)$. The composite was made by mixing the following material: $\mathrm{B} / \mathrm{H}$ resin mixture $(25 \%), 70 \%$ strontium fluoroaluminasilicate glass powders and $5 \%$ silanized HL containing microcapsules. This material was mixed by a speed mixer (DAC 150 FVZ, FlackTek, Landrum, SC, USA) at 3500 RPM for 1 minute, and then hand mixed carefully. The mixing process was repeated for 3 times until the mixture becomes a uniformly mixed clay-like substance. The specimens were fitted into metal molds of the required 
dimensions, and photo-cured using a Triad 2000 Light-curing Unit (Dentsply, York, PA, USA) with a tungsten halogen light bulb $\left(75 \mathrm{~W}\right.$ and $\left.120 \mathrm{~V}, 43 \mathrm{~mW} / \mathrm{cm}^{2}\right)$ for $2 \mathrm{~min}$ each from both open sides of the assembly. The hardened composite was then removed from the mold and polished by sandpaper until its surface become smooth. Variations in the material was done by increasing the wt $\%$ of microcapsules and reducing the wt $\%$ of the strontium fluoroaluminosilicate glass powders. The wt $\%$ of the resin was unchanged. All specimen used for elastic modulus and fracture toughness measurement were left for 24 hours after initial curing before mechanical testing.

Elastic modulus measurement: Elastic modulus was determined according to ISO4049: 2009. Five bars (2 mm x $2 \mathrm{~mm} \times 25 \mathrm{~mm})$ of each composition were made following the SHDC construction method descripted above. Elastic modulus was measured using a Dynamic Mechanical Analysis (DMA Q800, TA Instruments, DE, USA) set with a loading rate of 1 $\mathrm{N} / \mathrm{min}$. The specimens were placed on a 3-point bending test device, which was constructed with $20 \mathrm{~mm}$ span distance between supports and ensuring an equally distributed load. The elastic modulus of each material was calculated according to ISO4049: 2009.

Characterization of healing based on mechanical, morphological and chemical evaluation: Mechanical evaluation was carried out through fracture toughness, which is determined according to ISO6872: 2014. Five bars (25 mm x $4 \mathrm{~mm}$ x $3 \mathrm{~mm}$ ) of each composition were made following the SHDC construction method descripted above. The specimens were notched by sawing with an IsoMet Low Speed Saw (Buehler, Lake Bluff, IL, USA) fitted with NTI Flex Diamond Discs (NTI-Kahla GmbH, Kahla, Germany), and the notch was sharpened using a razor blade polished with $3 \mu \mathrm{m}$ diamond paste. Fracture toughness was assessed using an Instron 5500R Universal Testing Machine (Instron Corp., Canton, MA, USA) set with a loading speed of $0.5 \mathrm{~mm} / \mathrm{min}$. The specimens were placed on a 3-point bending test device, which was constructed with $20 \mathrm{~mm}$ span distance between supports and ensuring an equally distributed load. The specimen was loaded until critically fractured, and 
the load was immediately stopped to recover the specimen. A drop of water was added into the notch of the specimen, and the specimen was rested for 4 days. The process was then repeated to measure the peak load of the healed specimen. A Helios NanoLab 650 Dual Beam SEM/FIB (FEI Company, Hillsboro, Oregon, USA) with an X-Max $80 \mathrm{~mm}^{2}$ SDD-EDS detector (Oxford Instruments, Abingdon, Oxfordshire, UK) was used to analyze the elemental composition of the fractured and healed surfaces.

Statistical analysis. The elastic modulus, fracture toughness, and size of microcapsules were analyzed using one-way analysis of variance (ANOVA) with a $95 \%$ confidence interval to indicate significant differences.

\section{Results}

\subsection{Microencapsulation of healing liquid (HL)}

The formation of silica microcapsules and encapsulation of HL (an aqueous solution of polyacid) was confirmed by scanning electron microscopy (SEM) and FTIR (Figure 2A). Microcapsules containing methylene blue (MB) aqueous solution were also prepared, and used as a control. The average diameter of the microcapsules was $29.46 \mu \mathrm{m} \pm 10.28 \mu \mathrm{m}$ based on 200 microcapsules in twelve SEM images, with a thickness ranging from $4-8 \mu \mathrm{m}$; microcapsules that were over $100 \mu \mathrm{m}$ in diameter with thickness of approximately $20 \mu \mathrm{m}$ were also observed in the SEM images. FTIR spectra from HL-containing microcapsules indicated the presence of $-\mathrm{COOH}$ groups (Figure 2A) with an absorbance peak at $1730 \mathrm{~cm}^{-1}$; FTIR spectra from the MB control microcapsules did not show this peak.

The mass fraction of polyacid in the encapsulated HL was determined by TGA (Figure 2B). The temperature program was divided into two region to separate water and other hightemperature volatiles. Mass loss below $150{ }^{\circ} \mathrm{C}$ was treated as the result of water evaporation, and above $150{ }^{\circ} \mathrm{C}$ was the consequence of losing polyacids and other materials in the microcapsules. Water-containing microcapsules ( $\mathrm{HCl}$ solutions were actually encapsulated) 
were used as a baseline to correct these non-polyacid vaporized and burned at high temperature. The water content in HL-containing microcapsules was $30.2 \%$. The mass change of HL-containing microcapsules at high temperature range was $11.1 \%$, and it was $4.2 \%$ for water-containing microcapsules in that range. Overall, the mass fraction of polyacid in HL-containing microcapsules was approximately $17 \%$.
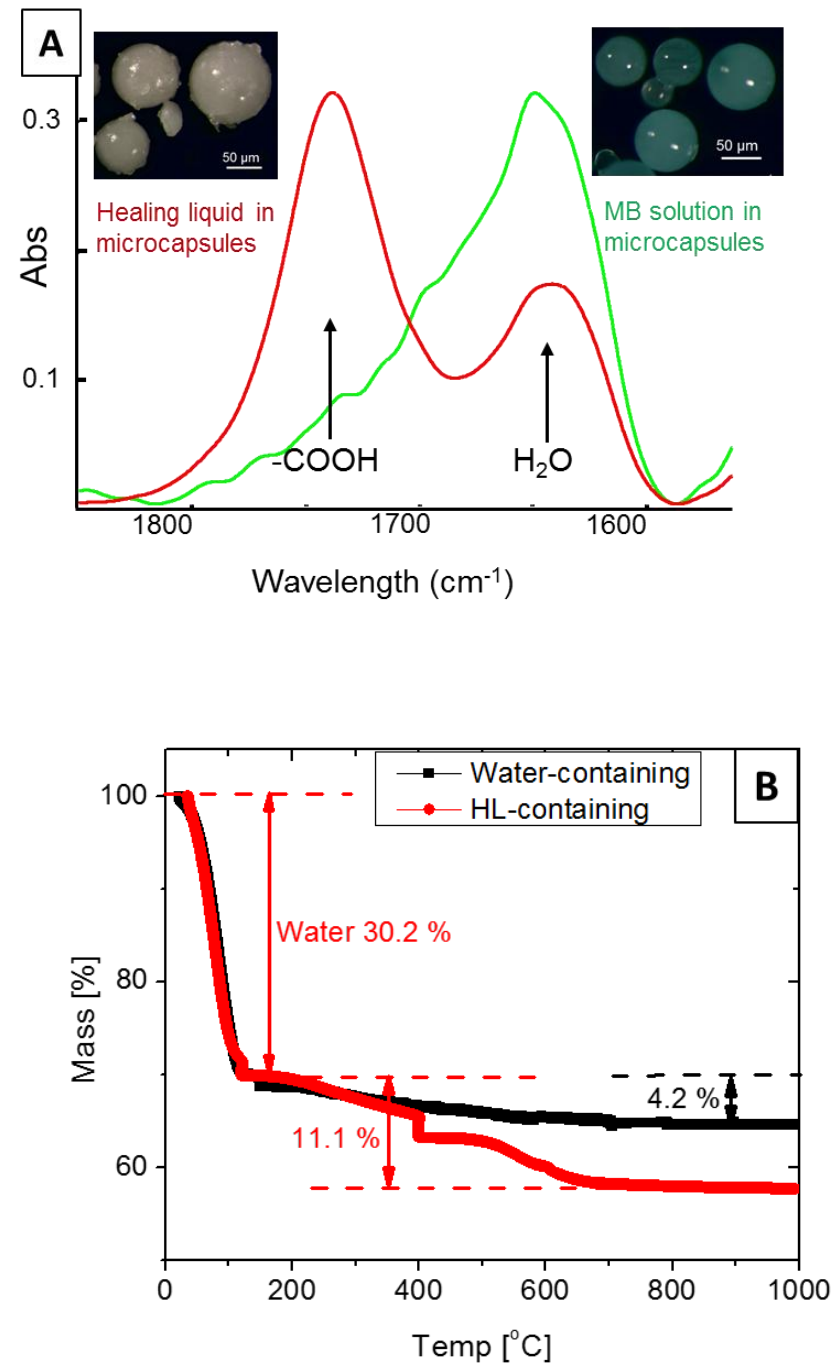

Figure 2. Characterization of microcapsules. (A) FTIR spectra of the microcapsules, indicating the presence of polyacid in HL or methylene blue aqueous solutions; and (B) TGA of water-containing microcapsules and HL-containing microcapsules

\subsection{Self-Healing dental composite (SHDC) construction}

Silanization of microcapsules improved the efficiency of capsule fracture triggered by the cracks. An SEM image of the cross-section of a SHDC made with unsilanized microcapsules is shown in Figure 3A. Figure 3B shows the cross-section of a SHDC containing silanized 
microcapsules. In Figure 3A, the wall of a fractured microcapsule is distinctly separated from the resin matrix and fillers. The morphology of materials (dried HL) inside the microcapsule is different from the rest in the image. In Figure 3B, it is hard to distinguish the walls of silanized mircocapsules from the resin matrix and fillers. The existence of half-spheres suggests the presence of fractured microcapsules. When silane agents were applied, approximately $72 \%$ of microcapsules at the fracture surface of composites were broken based on SEM image analyses. In contrast, when the microcapsules were not treated with the silane agent, about $15 \%$ of the microcapsules were broken, and this only happened to super-large microcapsules, over 100 um in diameter (Figure 3A).
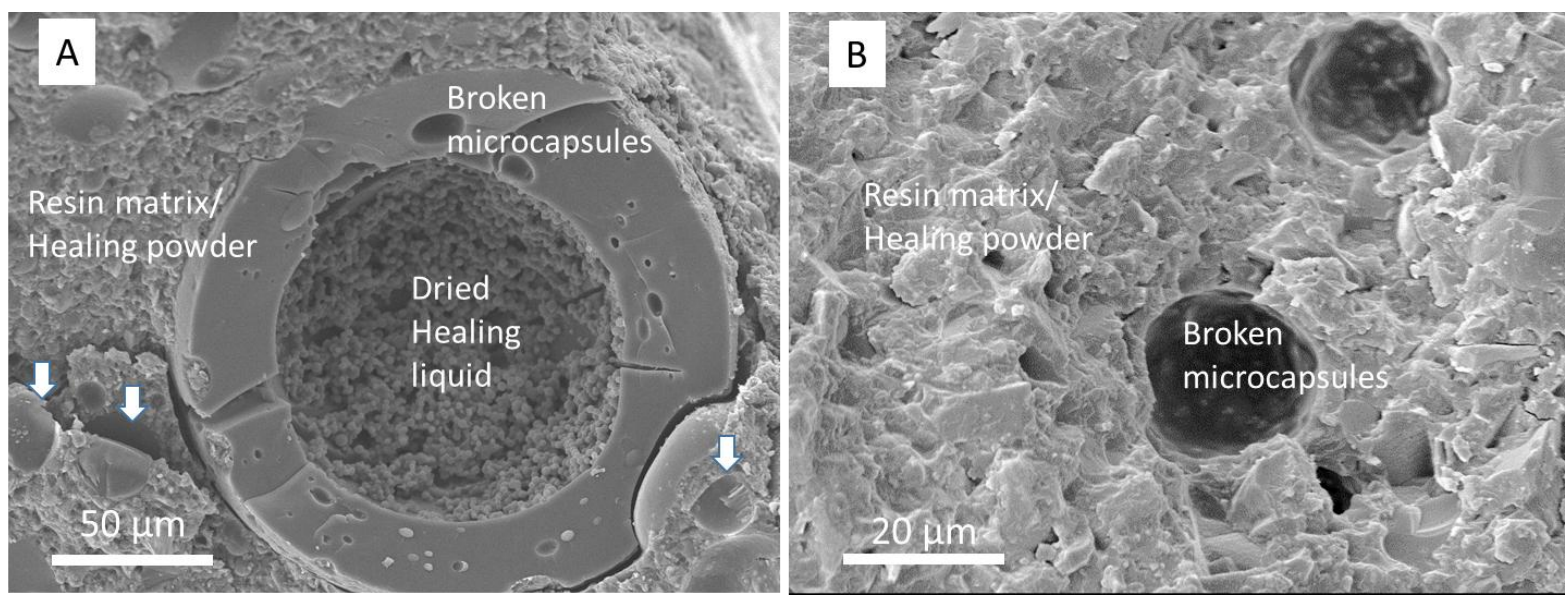

Figure 3. SEM images of SHDC cross-sections. (A) A cross-section of SHDC with unsilanized microcapsules. The components of SHDC are labeled, and the arrows point to pits left by microcapsules; (B) a cross-section of SHDC with silanized microcapsules.

\subsection{Elastic modulus}

The mechanical tests in this study were all carried out $1 \mathrm{~d}$ after light irradiation, to be consistence with the methods previously used in our group. [31, 32] The elastic moduli of SHDCs with different mass percentages of microcapsules were assessed, and the results are shown in Figure 4. Composites that consisted of the same mass percentages of watercontaining microcapsules were used as controls, and their elastic moduli are also shown in Figure 4. Based on one-way ANOVA analysis, the SHDCs had the same moduli as that of their controls. Increasing the mass fraction of microcapsules slightly reduced the elastic 
modulus. Statistically, the elastic moduli of SHDCs with $2.5 \mathrm{wt} \%$ of microcapsules and 5.0 wt $\%$ of microcapsules were the same. The elastic moduli of SHDCs with $10 \mathrm{wt} \%$ of microcapsules was significantly lower than the rest compositions $(\mathrm{p}<0.05)$.

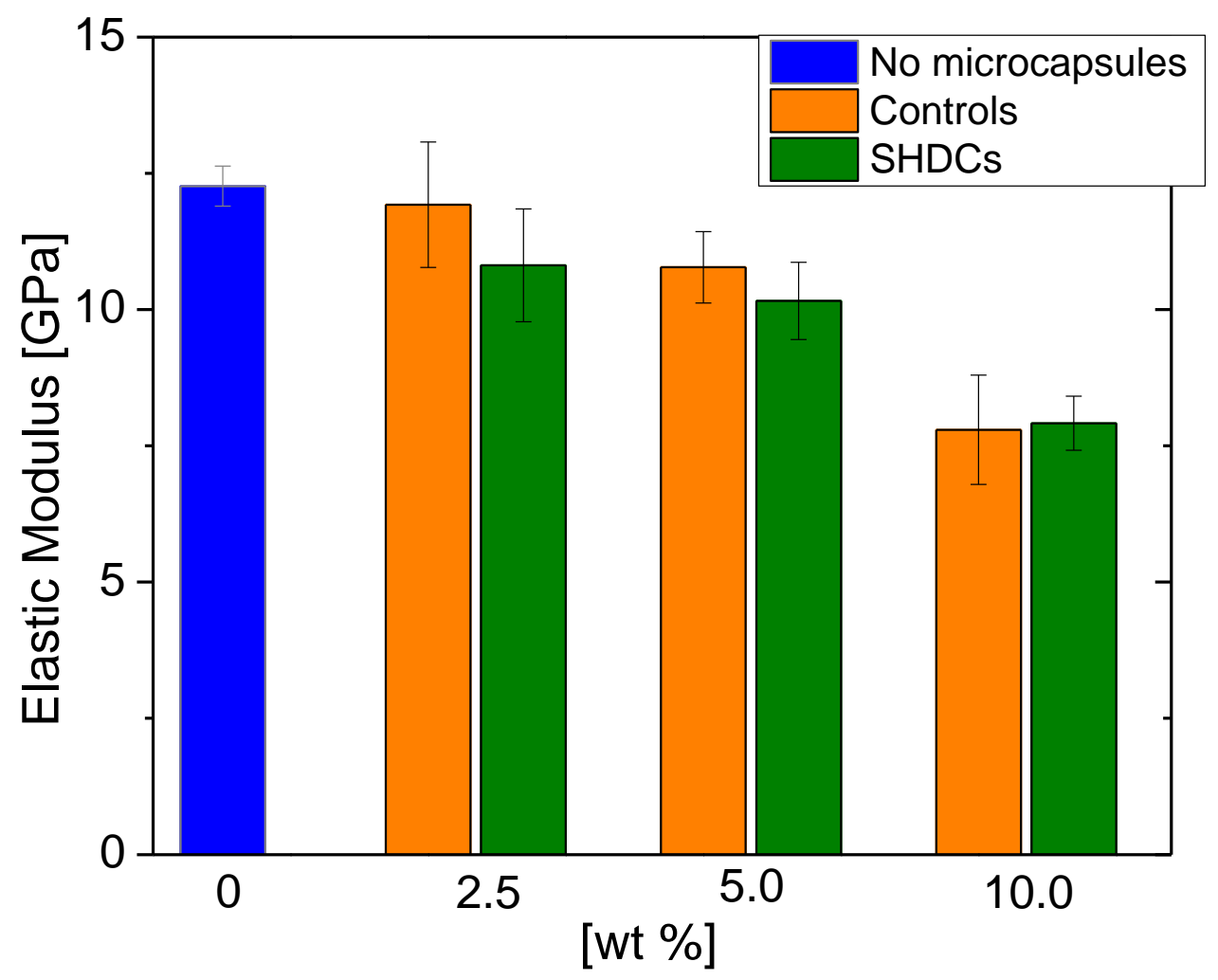

Figure 4. Elastic moduli of SHDCs and control composites containing different mass percentages of microcapsule

\subsection{Fracture toughness and healing efficiency}

The initial fracture toughness $\left(K_{I C}{ }^{i n i}\right)$ of SHDCs at different mass percentages of microcapsules and their fracture toughness after healing $\left(K_{I C}{ }^{\text {heal }}\right)$ are listed Table 1 . The controls were the same as those used for elastic modulus measurement. The fracture toughness was obtained using a single edge V-notched beam (SEVNB) method based on the peak load to fracture the specimens. The dimensions of specimens for SEVNB tests are listed in Figure 5A. An example of the notch and the fracture of a real specimen before and after fracture are shown in Figure 5B and Figure 5C, respectively. 
The SEVNB method induced a controlled cracking in the specimen and, if the load on the specimen was precisely stopped, can critically fracture the specimen without severing it into two pieces. The initial fracture toughness $\left(K_{I C}^{i n i}\right)$ was calculated according to Equation 1. Water was added into the notch, and the specimem was held together for four days. The specimen was fractured again using the same method, and the new fracture toughness $\left(K_{I C}{ }^{\text {heal }}\right)$ was calculated. The healing efficiency is defined as the percentage of the new fracture toughness recovered in comparison with the original fracture toughness $\left(K_{I C}^{\text {heal }} / K_{I C}^{\text {ini }}\right.$ $* 100 \%)$. A set of non-healing composites with the same mass percentage of water-filled microcapsules were used as the controls.

$$
K_{I c}=g\left[\frac{F_{\max } S_{0} \times 10^{6}}{B W^{1.5}}\right]\left[\frac{3(a / W)^{1.5}}{2(1-a / W)^{1.5}}\right]
$$

Equation 1. The equation for calculating fracture toughness $K_{I c}$ using a three-point fixture, where $S_{0}=$ span, $W=$ thickness, $B=$ width, $a=$ notch depth, $F_{\max }=$ fracture load, and $g=A_{0}$ $+A_{1}(a / W)+A_{2}(a / W)^{2}+A_{3}(a / W)^{3}+A_{4}(a / W)^{4}+A_{5}(a / W)^{5}$. The coefficients to calculate the polynomial $g$ when $S_{0} / W=5$ are: $A_{0}=1.9109, A_{1}=-5.1552, A_{2}=12.6880, A_{3}=-19.5736, A_{4}$ $=15.9377, A_{5}=-5.1454$

The $K_{I C}{ }^{i n i}$ of SHDCs and the controls was the same when the same mass percentage of microcapsules was used. The $K_{I C}{ }^{i n i}$ did not change when up to 5 wt $\%$ of microcapsules were added. Further increase in the microcapsule loading decreased the $K_{I C}{ }^{i n i}$. The $K_{I C}{ }^{i n i}$ values of SHDCs with 10 wt $\%$ of microcapsules were lower than those of the other compositions.

The SHDCs with 5 wt $\%$ and 10 wt $\%$ of microcapsules all showed healing capabilities while their controls did not. According to one-way ANOVA, the healing efficiency was statistically the same for SHDCs with these two mass percentages of microocapsules. The average healing efficiency was approximately $25 \%$ (Table 1). As an example, Figure 6 lists the stress-strain curves of a 5 wt $\%$ SHDC and a control composite before and after healing. These two composites required the same load to fracture initially, and this intial load is recovered partially in SHDC, but not in the control. 


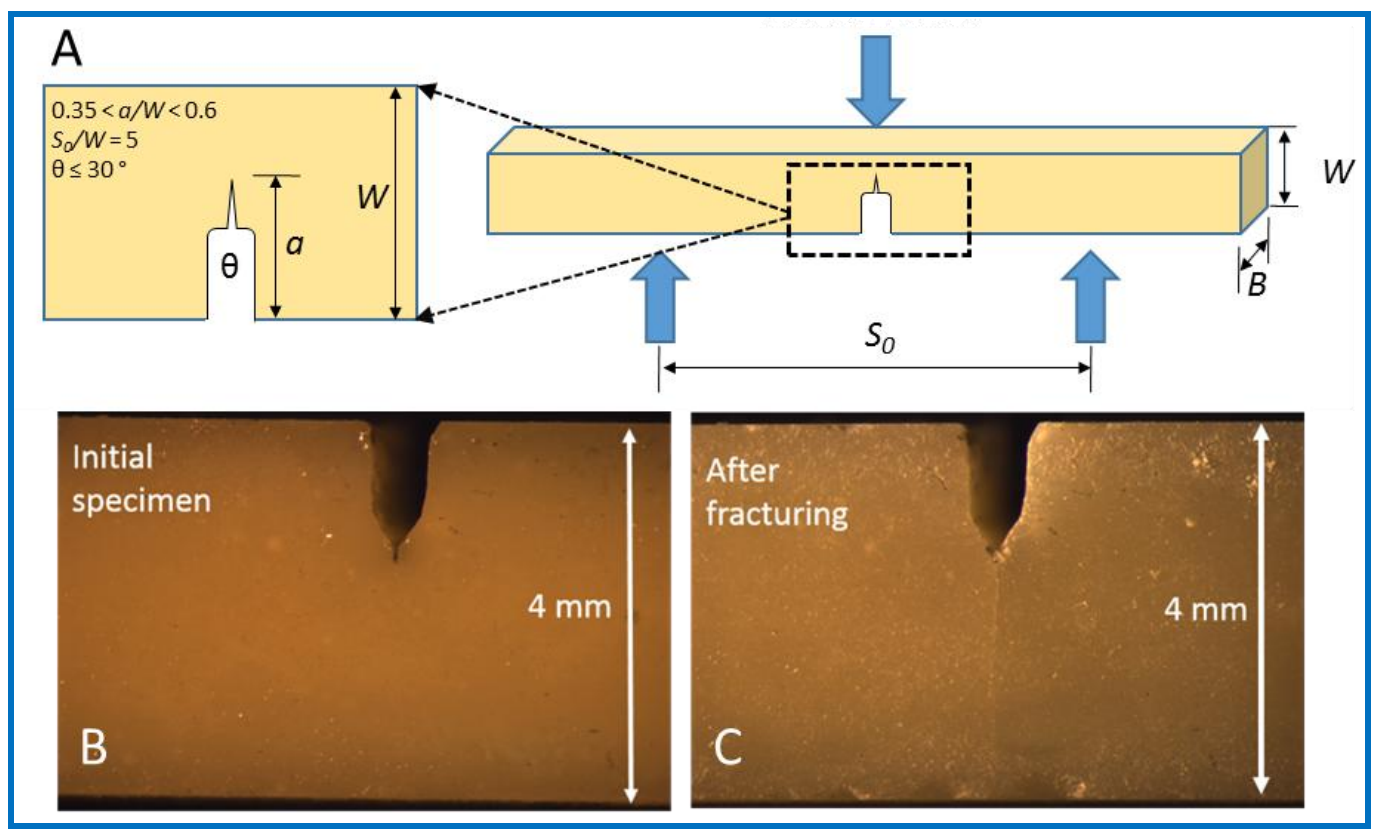

Figure 5. (A) A model of the SEVNB process indicates the required geometry for the Vnotch: $S_{0}=20 \mathrm{~mm}, W=4 \mathrm{~mm}$, and $B=3 \mathrm{~mm}$; and the load is applied from the center of the bar. (B) A stereo microscope image shows the notched specimen before fracture, and (C) a stereo microscope image shows the same specimen after reached the fracture load.

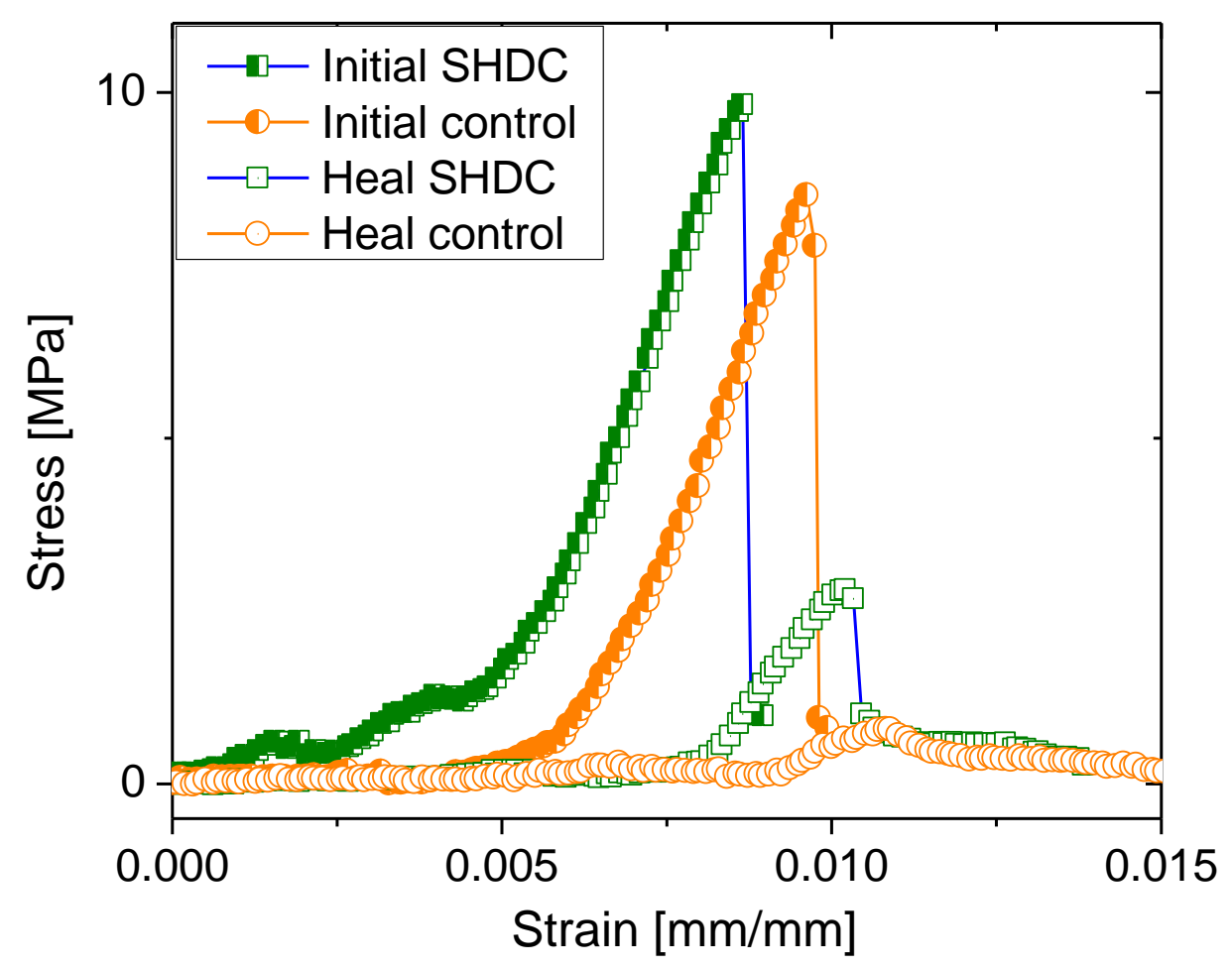

Figure 6. Stress-strain curves of a SHDC having 5 wt \% of microcapsules before and after healing, in comparison to a control composed with the same mass fraction of water-containing microcapsules. 
Table 1. Fracture toughness and healing efficiency of SHDCs and control composites

\begin{tabular}{ccccc}
\hline $\begin{array}{c}\text { wt \% of } \\
\text { microcapsules }\end{array}$ & $\begin{array}{c}\text { Material } \\
\text { encapsulated }\end{array}$ & $\begin{array}{c}K_{I C}{ }^{\text {ini }} \\
{\left[\mathrm{MPa} \cdot \mathrm{m}^{1 / 2}\right]}\end{array}$ & $\begin{array}{c}K_{I C}^{\text {heal }} \\
{\left[\mathrm{MPa} \cdot \mathrm{m}^{1 / 2}\right]}\end{array}$ & $\begin{array}{c}\text { healing efficiency } \\
{[\%]}\end{array}$ \\
\hline $0 \%$ & No & $0.92 \pm 0.02$ & -- & -- \\
$2.5 \%$ & Water & $0.91 \pm 0.07$ & $0.07 \pm 0.05$ & $7.0 \pm 6.0$ \\
$2.5 \%$ & $\mathrm{HL}$ & $0.90 \pm 0.04$ & $0.08 \pm 0.02$ & $9.1 \pm 2.1$ \\
$5 \%$ & Water & $0.95 \pm 0.04$ & $0.11 \pm 0.04$ & $11.6 \pm 3.4$ \\
$5 \%$ & $\mathrm{HL}$ & $0.91 \pm 0.03$ & $0.22 \pm 0.03$ & $24.2 \pm 3.8$ \\
$10 \%$ & Water & $0.85 \pm 0.11$ & $0.08 \pm 0.05$ & $9.8 \pm 6.5$ \\
$10 \%$ & $\mathrm{HL}$ & $0.82 \pm 0.06$ & $0.21 \pm 0.06$ & $25.4 \pm 7.0$ \\
\hline
\end{tabular}

3.5. Morphological and chemical observation of healing

The morphological and chemical changes after healing were confirmed by SEM equipped with Energy dispersive X-ray spectroscopy (EDS). A new material was formed on the cross section of the specimen after the healing process was completed. This material had a different appearance to the non-healed fracture surface of the SHDC under SEM (Figure 7A and 7B). Based on our self-healing model, this new material was expected to be GIC, the reaction product of the strontium fluoroaluminosilicate particles (HP) and the HL. EDS confirmed the chemical transition from HP to GIC at the healed fracture surface. As the key elements in the formation of GIC, the elements $\mathrm{Al}, \mathrm{Si}$ and $\mathrm{Sr}$ were identified and evaluated. In sections before healing (Figure 7C and 7E), the Si content is higher than $\mathrm{Al}$, while in the healed regions (Figure 7D and 7F) the content of $\mathrm{Al}$ and $\mathrm{Sr}$ increased. Furthermore, the healed surface had an aluminum-silicon-strontium ratio of $0.96: 1: 0.41$, matching that in GIC powder and GIC (Table 2).

Table 2. EDS measured values of the aluminum-silicon-strontium ratio from the surfaces of SHDC with $5 \%$ microcapsules.

\begin{tabular}{lccccc}
\hline Sample & $\begin{array}{c}\mathrm{Al} \\
\text { (atomic \%) }\end{array}$ & $\begin{array}{c}\mathrm{Si} \\
\text { (atomic \%) }\end{array}$ & $\begin{array}{c}\mathrm{Sr} \\
\text { (atomic \%) }\end{array}$ & Al-Si ratio & Sr-Si ratio \\
\hline Non-healed section & $31.5 \pm 6.9$ & $54.9 \pm 4.6$ & $13.6 \pm 2.9$ & $0.57: 1$ & $0.25: 1$ \\
Healed section & $40.5 \pm 1.3$ & $42.1 \pm 1.3$ & $17.4 \pm 1.1$ & $0.96: 1$ & $0.41: 1$ \\
GIC powder & $41.3 \pm 2.4$ & $41.1 \pm 6.0$ & $17.7 \pm 0.9$ & $1.00: 1$ & $0.43: 1$ \\
GIC & $42.3 \pm 1.3$ & $42.3 \pm 2.0$ & $15.4 \pm 1.5$ & $1.00: 1$ & $0.36: 1$ \\
\hline
\end{tabular}




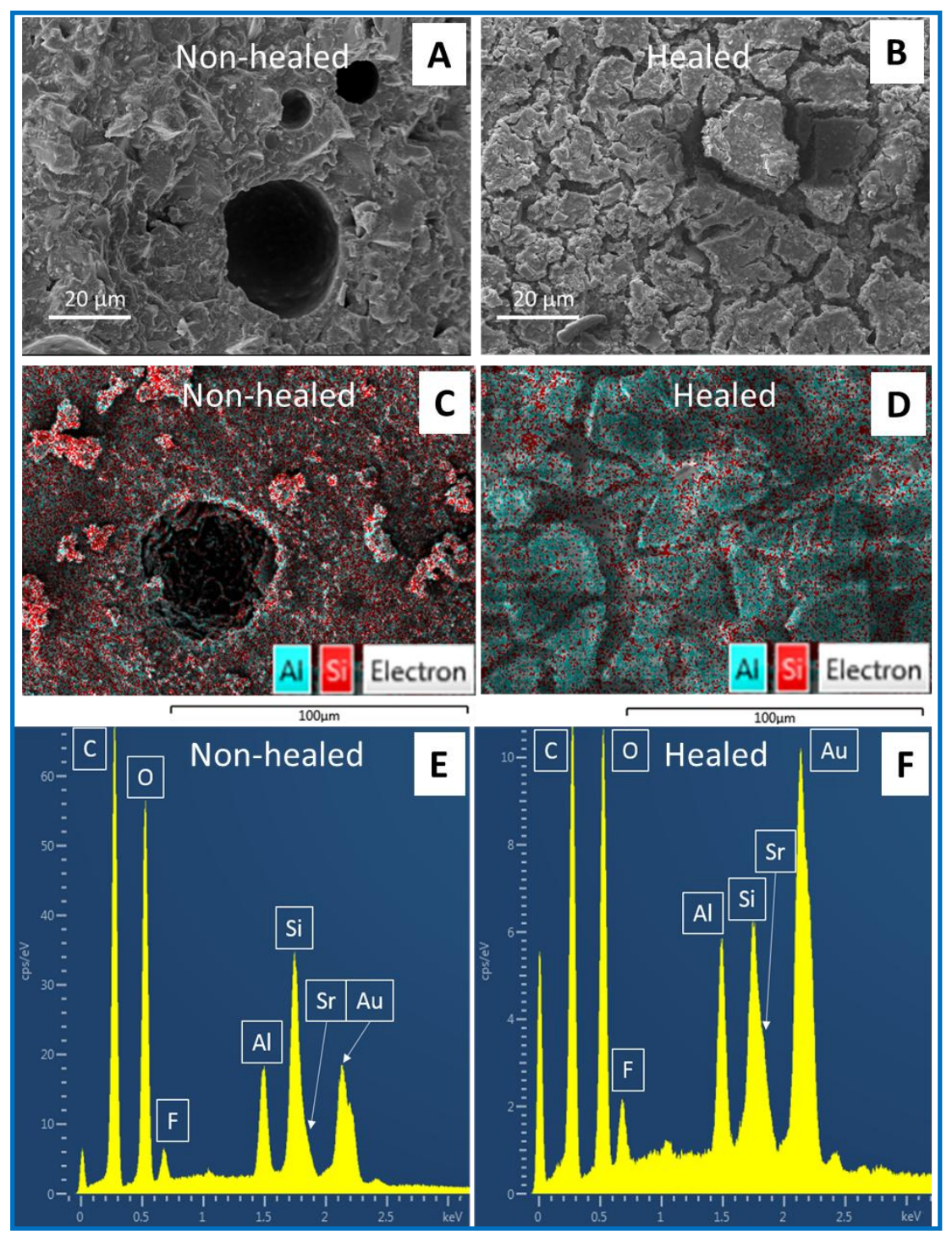

Figure 7. SEM images of the (A) non-healed SHDC surface, and (B) the healed SHDC surface. SEM-EDS images of (C) non-healed SHDC surface, and (D) the healed SHDC surface, artificially colored to match the signal count of $\mathrm{Al}$ and $\mathrm{Sr}$; and the elemental analysis by EDS on sections of the (E) non-healed, and (F) healed regions of the SHDC.

\section{Discussion}

Encapsulation of the HL in microcapsules was one of the keys to successful SHDCs. Silica microcapsules were used because they had the elastic modulus matching the fillers in the dental composites, which prevented premature fracture during composite preparation. [33] In addition, the acidic and hydrophilic nature of the HL required an identical medium during encapsulation process. The encapsulation of HL was achieved by making a water/oil emulsion first, where the HL was in the aqueous phase. Tetraethyl orthosilicate (TEOS) and HCl were 
mixed. After the mixture ( $\mathrm{pH}=0.6$ to 1 ) reached its stability, it was added to the emulsion. Upon contacting with water in low $\mathrm{pH}$, TEOS went through a condensation reaction to form a silica network. This silica network surrounded the aqueous droplet and eventually formed spherical capsules, which captured the desired water-soluble reagents, including HL, from the water/oil mixture. The size of the microcapsules was determined by stirring speed and surfactants used to make emulsions, and the rate of this encapsulation process was controlled through acidity (which catalyzes TEOS condensation) and temperature. [29, 30] With this process, we successfully encapsulated $\mathrm{HLs}$ and $\mathrm{HCl}$ solutions. The HL-containing microcapsules were used in SHDCs, and water-containing ( $\mathrm{HCl}$ solutions) microcapsules were incorporated in the control composites.

Silanization was another key for successful SHDCs, which ensured the fracture of microcapsules in response to crack propagation and delivery of the HL. Silane agents provide significant improvement in the fracturing of microcapsules and the releasing of the HL.

Dental resin composites and GIC are two types of dental restorative materials. In addition, resin modified GIC (RM-GIC) and compomers (polyacid-modified composite) are also used in dental clinics. [34-36] The SHDC is designed to have the same basic structure as clinically tested dental composites. The resin monomers used in this composite were Bis-GMA and HEMA; both of which are commonly used in dental composites. HEMA is also the most often used monomer in RM-GIC; [37, 38] this is due to its hydrophilicity, which allows the movement of water required by the GIC reaction. Under a more strict classification, the SHDC we developed is actually a compomer. [39, 40] The compomers contain a fluoridereleasing silicate that reacts with polyacids to release fluoride. In SHDCs, the polyacid is incorporated to form GIC, and achieves autonomous healing of cracks. Fluoride-release is not the primary aim of this study, although it is an important beneficial property for dental restorative materials $[35,36,41]$ to prevent secondary caries. 
The mechanical properties of the SHDCs make them suitable for use as dental restorative materials. The modulus and fracture toughness values in SHDCs are stronger than those in GICs and most of the RMGICs, and close to compomers and resin composites. The mechanical performance of the SHDC still needs to be improved, which could be achieved through, but not limited to, the following modifications: use of a tougher resin network, addition of nanoparticles, and optimization of the size of the microcapsules.

The addition of an excess of microcapsules, and especially the presence of super-large microcapsules, reduced the elastic modulus. The super-large microcapsules become "weak spots" that compromise the mechanical performance of the SHDCs. [33, 42, 43] This is consistent with the healing material developed using the monomer-catalyst model, [21] where the increase of microcapsule loading was a tradeoff of mechanical performance for improved healing.

In comparison with the SHDC, the controls had the same elastic modulus, and fracture toughness. For calculating healing efficiency, a method used in the UIUC models was adopted. $[21,44]$ This method involves stressing the sample with a load that induces internal fracturing, allowing it to rest for several days, and then measuring the new peak load after healing. Specimens that were V-notched did not break into two pieces at the peak load because the crack growth was stopped to keep the two halves slightly connected. This maintained the two pieces perfectly matched during the healing process. The crack was then healed by adding water into the notch, allowing it to assist the diffusion of the HL, much like what would happen in the oral environment. After a period of four days, $K_{I C}^{\text {heal }}$ was determined. The maximum healing efficiency of one SHDC specimen reached up to $35 \%$ when $10 \mathrm{wt} \%$ of microcapsules were used. The healing efficiency can be improved through optimizing the composition of the SHDC, including a more proficient HL delivery mechanism, a more effective reaction between HP and HL, better mechanical performance of the formed GIC 
(making the new GIC more difficult to fracture), and improved integration of GIC and resin matrix. [45-47]

Morphologic and chemical changes observed at the fracture surface also confirmed the reaction of HP and HL, and the formation of GIC. SEM-EDS revealed that the microcapsules were fractured; the HP disappeared; and a layer of new materials formed. This layer of material was similar to GIC, as designed, based on aluminum-silicon-strontium ratio determined by EDS. On a non-healed section of SHDC, the Al : Si ratio was $0.57: 1$, which had a higher Si element count than that in the HP, due to the addition of silica microcapsules in the SHDC. On a healed section with a layer of new materials, the Al : Si ratio was $0.96: 1$. The increased aluminum content was rationalized by the nature of the GIC reaction. The polyacid in HL reacted with the Lewis basic salts in the HP, dissolved the HP, and produced strontium and aluminum polyacrylates. These polyacrylates formed a new network and covered the fracture surface. In a healed section, more aluminum and strontium elemental signals were detected than those on the non-healed surface. The dissolving process of HP by HL produced physical interlocking between the new GIC and the resin network. This interlocking is important in repairing the cracks and increasing the service life of the composites.

All of the materials used in this SHDC model have been used as parts of dental restorative materials. They have been serving in dental clinics for several decades. [25, 27] To the best of our knowledge, this SHDC is the first successful self-healing dental composites that contained only clinically tested materials. Using the GIC liquid and powder as healing reagents heals cracks autonomously and completely replaces the potentially toxic monomers and pricy initiators, such as dicyclopentadiene and ruthenium catalysts. In addition, the great flexibility and clinical efficacy of GICs justify the utilization of SHDCs as medical devices. [48, 49] The GICs are biocompatible, inherently adhere to tooth structure, exhibit little or no shrinkage, and release $F^{-}$to prevent and/or inhibit caries. [41, 50] Additional functionality of SHDCs can 
be achieved by adding other active components to the HL in the microcapsules or by mixing them with HP. Water based antimicrobial agents such as chlorhexidine (used in mouthwashes to reduce dental plaque and oral bacteria) [51] can be incorporated into microcapsules with the HL and provide instant protection against bacterial attack. Moreover, a portion of HP can be replaced with hydroxyapatite to improve tooth remineralization. [52, 53]

\section{Conclusion}

A self-healing dental composite (SHDC) model was developed and evaluated. The SHDCs contained HP and HL encapsulated in silica microcapsules, in addition to resin network and fillers. A set of GIC powder and GIC liquid was used as HP and HL, respectively. The keys to achieve autonomous healing and prepare clinically applicable dental restorative composites, such as encapsulation of HL, microcapsule preparation and silanization, were discussed. The elastic modulus and fracture toughness of model SHDCs were evaluated, which supported the use of SHDCs as dental restorative materials. The success of crack healing was confirmed based on the recovery of fracture toughness of the SHDCs in terms of healing efficiency. Average healing efficiency of SHDCs reached up to $25 \%$, which confirmed an effective healing achieved in this SHDC. Combining the performance in elastic modulus and fracture toughness, the SHDCs with 5 wt $\%$ of microcapsules had the best overall performance against the other two compositions. In addition, morphological and chemical observation of the healed cracks supported the proposed 3-step healing procedure: fracture, deliver and heal. Furthermore, this model achieved autonomous healing of cracks with materials being used in dental clinics, which is an advantage to accelerate the technology transition from lab to dental clinics.

\section{Acknowledgements}

This work was funded by the National Institute of Dental and Craniofacial Research (U01DE023752). Financial support was also provided through the ADA Foundation. The authors would like to thank Dr. Rafael Bowen, Mr. Anthony Giuseppetti, and Mr. George 
Quinn for their technical recommendations. We also would like to thank Center for Nanoscale Science and Technology (CNST) at NIST for their technical support. 


\section{References}

[1] Brochu ABW, Craig SL, Reichert WM. Self-healing biomaterials. Journal of Biomedical Materials Research Part A. 2011;96A:492-506.

[2] Esser-Kahn AP, Thakre PR, Dong HF, Patrick JF, Vlasko-Vlasov VK, Sottos NR, et al. Three-dimensional microvascular fiber-reinforced composites. Advanced Materials.

2011;23:3654-8.

[3] Hager MD, Greil P, Leyens C, van der Zwaag S, Schubert US. Self-Healing Materials. Advanced Materials. 2010;22:5424-30.

[4] Diesendruck CE, Sottos NR, Moore JS, White SR. Biomimetic Self-Healing. Angewandte Chemie-International Edition. 2015;54:10428-47.

[5] Singh AV, Rahman A, Kumar N, Aditi AS, Galluzzi M, Bovio S, et al. Bio-inspired approaches to design smart fabrics. Mater Des. 2012;36:829-39.

[6] Zhao N, Wang Z, Cai C, Shen H, Liang FY, Wang D, et al. Bioinspired Materials: from Low to High Dimensional Structure. Advanced Materials. 2014;26:6994-7017.

[7] Dry C. Procedures developed for self-repair of polymer matrix composite materials. Compos Struct. 1996;35:263-9.

[8] White SR, Sottos NR, Geubelle PH, Moore JS, Kessler MR, Sriram SR, et al. Autonomic healing of polymer composites. Nature. 2001;409:794-7.

[9] Ferracane JL. Resin composite-State of the art. Dental Materials. 2011;27:29-38.

[10] Jandt KD, Sigusch BW. Future perspectives of resin-based dental materials. Dental Materials. 2009;25:1001-6.

[11] Hillewaere XKD, Du Prez FE. Fifteen chemistries for autonomous external self-healing polymers and composites. Prog Polym Sci. 2015;49-50:121-53.

[12] Zhu DY, Rong MZ, Zhang MQ. Self-healing polymeric materials based on microencapsulated healing agents: From design to preparation. Prog Polym Sci. 2015;4950:175-220.

[13] Lehn JM. Perspectives in Chemistry-Aspects of Adaptive Chemistry and Materials. Angewandte Chemie-International Edition. 2015;54:3276-89.

[14] Van Tittelboom K, De Belie N. Self-Healing in Cementitious Materials-A Review. Materials. 2013;6:2182-217.

[15] Aissa B, Therriault D, Haddad E, Jamroz W. Self-Healing Materials Systems: Overview of Major Approaches and Recent Developed Technologies. Adv Mater Sci Eng. 2012.

[16] Mauldin TC, Kessler MR. Self-healing polymers and composites. Int Mater Rev. 2010;55:317-46.

[17] Olugebefola SC, Aragon AM, Hansen CJ, Hamilton AR, Kozola BD, Wu W, et al. Polymer Microvascular Network Composites. J Compos Mater. 2010;44:2587-603.

[18] Jones AS, Rule JD, Moore JS, Sottos NR, White SR. Life extension of self-healing polymers with rapidly growing fatigue cracks. J R Soc Interface. 2007;4:395-403.

[19] Jones AS, Rule JD, Moore JS, White SR, Sottos NR. Catalyst morphology and dissolution kinetics of self-healing polymers. Chem Mat. 2006;18:1312-7.

[20] Ferracane JL. Resin-based composite performance: Are there some things we can't predict? Dental Materials. 2013;29:51-8.

[21] Wu JL, Weir MD, Melo MAS, Xu HHK. Development of novel self-healing and antibacterial dental composite containing calcium phosphate nanoparticles. Journal of Dentistry. 2015;43:317-26.

[22] Ouyang XB, Huang XQ, Pan QH, Zuo CQ, Huang C, Yang XL, et al. Synthesis and characterization of triethylene glycol dimethacrylate nanocapsules used in a self-healing bonding resin. Journal of Dentistry. 2011;39:825-33. 
[23] Wertzberger BE, Steere JT, Pfeifer RM, Nensel MA, Latta MA, Gross SM. Physical Characterization of a Self-Healing Dental Restorative Material. Journal of Applied Polymer Science. 2010;118:428-34.

[24] Lohbauer U. Dental glass ionomer cements as permanent filling materials? -Properties, limitations and future trends. Materials. 2010;3:76-96.

[25] Wilson AD, Kent BE. New translucent cement for dentistry-glass ionomer cement. Br Dent J. 1972;132:133-5.

[26] Wilson AD, Nicholson JW. Polyalkenoate cements. Chem Solid State Mater. 1993;3:90196.

[27] Bowen RL. Dental filling material. U.S. Dept. of Commerce . 1962. p. 3 pp.US ,066,112. [28] Bowen RL. Properties of a silica-reinforced polymer for dental restroations. Journal of the American Dental Association. 1963;66:57-64.

[29] Galgali G, Schlangen E, van der Zwaag S. Synthesis and characterization of silica microcapsules using a sustainable solvent system template. Mater Res Bull. 2011;46:2445-9. [30] Wang JX, Wang ZH, Chen JF, Yun J. Direct encapsulation of water-soluble drug into silica microcapsules for sustained release applications. Mater Res Bull. 2008;43:3374-81. [31] Sun JR, Forster AM, Johnson PM, Eidelman N, Quinn G, Schumacher G, et al. Improving performance of dental resins by adding titanium dioxide nanoparticles. Dental Materials. 2011;27:972-82.

[32] Sun JR, Fang R, Lin N, Eidelman N, Lin-Gibson S. Nondestructive quantification of leakage at the tooth-composite interface and its correlation with material performance parameters. Biomaterials. 2009;30:4457-62.

[33] Keller MW, Sottos NR. Mechanical properties of microcapsules used in a self-healing polymer. Exp Mech. 2006;46:725-33.

[34] De Munck J, Van Landuyt K, Peumans M, Poitevin A, Lambrechts P, Braem M, et al. A critical review of the durability of adhesion to tooth tissue: Methods and results. Journal of Dental Research. 2005;84:118-32.

[35] Nicholson JW. Polyacid-modified composite resins ("compomers") and their use in clinical dentistry. Dental Materials. 2007;23:615-22.

[36] Wiegand A, Buchalla W, Attin T. Review on fluoride-releasing restorative materials Fluoride release and uptake characteristics, antibacterial activity and influence on caries formation. Dental Materials. 2007;23:343-62.

[37] Berzins DW, Abey S, Costache MC, Wilkie CA, Roberts HW. Resin-modified Glassionomer Setting Reaction Competition. Journal of Dental Research. 2010;89:82-6.

[38] Geurtsen W. Biocompatibility of resin-modified filling materials. Crit Rev Oral Biol Med. 2000;11:333-55.

[39] Meyer JM, Cattani-Lorente MA, Dupuis V. Compomers: between glass-ionomer cements and composites. Biomaterials. 1998;19:529-39.

[40] Soncini JA, Maserejian NN, Trachtenberg F, Tavares M, Hayes C. The. longevity of amalgam versus compomer/composite restorations in posterior primary and permanent teeth Findings from the new England children's amalgam trial. Journal of the American Dental Association. 2007; 138:763-72.

[41] Aboush YEY, Torabzadeh H, Lee AR. 1-year fluoride release from fluoride-containing restorative materials. Journal of Dental Research. 1995;74:1.

[42] Kessler MR, Sottos NR, White SR. Self-healing structural composite materials. Compos Pt A-Appl Sci Manuf. 2003;34:743-53.

[43] BaLazs AC. Modelling self-healing materials. Materials Today. 2007;10:18-23.

[44] Aissa B, Therriault D, Haddad E, Jamroz W. Self-Healing Materials Systems: Overview of Major Approaches and Recent Developed Technologies. Adv Mater Sci Eng. 2012:17. 
[45] Moshaverinia A, Ansari S, Moshaverinia M, Roohpour N, Darr JA, Rehman I. Effects of incorporation of hydroxyapatite and fluoroapatite nanobioceramics into conventional glass ionomer cements (GIC). Acta Biomaterialia. 2008;4:432-40.

[46] Elsaka SE, Hamouda IM, Swain MV. Titanium dioxide nanoparticles addition to a conventional glass-ionomer restorative: Influence on physical and antibacterial properties. Journal of Dentistry. 2011;39:589-98.

[47] Moshaverinia A, Ansari S, Movasaghi Z, Billington RW, Darr JA, Rehman IU. Modification of conventional glass-ionomer cements with $\mathrm{N}$-vinylpyrrolidone containing polyacids, nano-hydroxy and fluoroapatite to improve mechanical properties. Dental Materials. 2008;24:1381-90.

[48] Frencken JE, van't Hof MA, van Amerongen WE, Holmgren CJ. Effectiveness of singlesurface ART restorations in the permanent dentition: A meta-analysis. Journal of Dental Research. 2004;83:120-3.

[49] Mickenautsch S, Yengopal V, Leal SC, Oliveira LB, Bezerra AC, Bonecker M. Absence of carious lesions at margins of glass-ionomer and amalgam restorations: a meta-analysis. Eur J Paediatr Dent. 2009;10:41-6.

[50] Mickenautsch S, Yengopal V, Banerjee A. Pulp response to resin-modified glass ionomer and calcium hydroxide cements in deep cavities: A quantitative systematic review. Dental Materials. 2010;26:761-70.

[51] Cao Z, Sun X, Yeh CK, Sun Y. Rechargeable Infection-responsive Antifungal Denture Materials. Journal of Dental Research. 2010;89:1517-21.

[52] Gu YW, Yap AUJ, Cheang P, Khor KA. Effects of incorporation of HA/ZrO2 into glass ionomer cement (GIC). Biomaterials. 2005;26:713-20.

[53] Zraikat H, Palamara JEA, Messer HH, Burrow MF, Reynolds EC. The incorporation of casein phosphopeptide-amorphous calcium phosphate into a glass ionomer cement. Dental Materials. 2011;27:235-43. 


\section{Graphical abstract:}

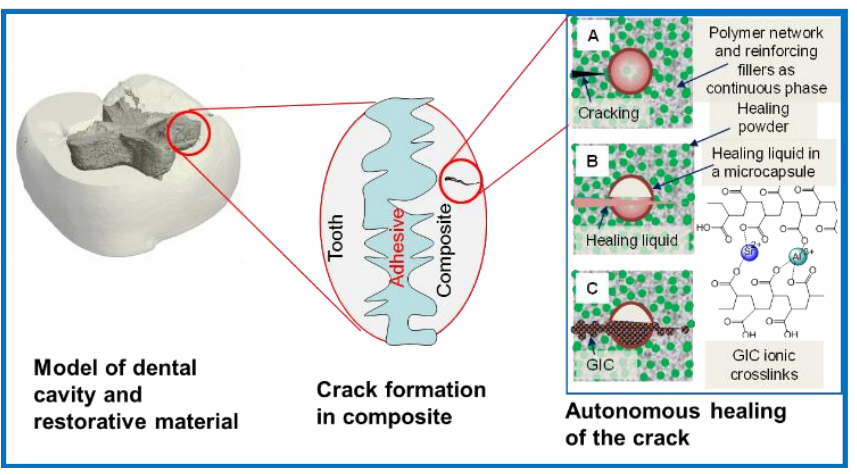

